\title{
Clipping and Filtering Technique for reducing PAPR In OFDM
}

\author{
Saleh Albdran ${ }^{1}$, Ahmed Alshammari ${ }^{2}$, Mohammad Matin ${ }^{3}$ \\ ${ }^{I}$ (Electrical \&Computer Engineering Department, University of Denver)
}

\begin{abstract}
Recently, Orthogonal Frequency Division Multiplexing (OFDM), as a multi-carrier, and a single carrier transmission systems are used widely in the communications industrial systems. Each one of these two techniques has their own advantages and disadvantages. To maintain a high data rate, OFDM is preferred over the single-carrier transmission system due to several shortages like, complexity of equalizing that increases proportionally with the data rate. To overcome this problem, OFDM is used as a transmission system with much better Bet Error Rate (BER) performance. Peak-to-Average-Power ratio (PAPR) is major drawback of using OFDM but we can deal with it using clipping and filtering as a power reduction technique.
\end{abstract}

Keywords-Additive White Gaussian Noise (AWGN), BER, high data rate, Multi-Carrier, OFDM, SingleCarrier (SC),

\section{INTRODUCTION}

During the last few years, the wireless communications went through several improvement stages in a fast way and as a result of that, the demand on the wireless services has growth rapidly as well. Due to the unpredictable nature of the wireless channels, calculating the propagation and the noise is not easy. On the other hand, the wired channels have less complexity of calculating the noise because that the signal propagates in a fixed path. On of the reasons to have signal degradation is the Additive White Gaussian Noise (AWGN) that could be occurred due to industrial or natural sources. There are also several types of fading channels include: multipath fading, frequency selective fading and others.

It is much easier to use single-carrier transmission scheme due to the simplicity and accuracy that is provided especially with the low data rate. This technique has its own advantages like the simplicity of transmitting the signal through a flat fading channel and saving more power since there is no need to extend the bandwidth by inserting guard interval. However, the use of single-carrier may have actual drawbacks with high data rate including equalizing complexity. Also Inter Symbol Interference (ISI) is a serious issue that appears with the multipath fading or frequency selective fading channels. OFDM is used to overcome the shortages of the single-carrier transmission scheme in the case of having high data rate [1]. The high bandwidth efficiency is one of the OFDM advantage in the case of having a big number of subcarriers. Also, with the OFDM scheme there is much lower chance to have ISI. Nevertheless, there are some OFDM drawbacks that could be the low bandwidth efficiency for having small subcarrier number. Also, we might have high peak values because of the IFFT operation in the transmitting side.

Frequency Division Multiplexing (OFDM), as a multiple carrier, outperforms the single carrier for communication systems mainly in high data rate. OFDM is getting so popular since it is less exposed to multipath effects, that makes it the core technique for the future standard systems, beside the high efficiency of its spectrum [2]. The main drawback of OFDM is the peak to average power ratio (PAPR) performance, which rises so high because of amplifiers nonlinear effects. The high PAPR essentially reduces the efficiency of the output power amplifier (PA). There are several techniques, to reduce the PAPR, which are divided into different categories: clipping technique, coding technique, probabilistic technique and so on [3] [4]. In this paper we will use clipping and filtering technique scheme to reduce the PAPR because of its easiness. However, this process could cause in-band distortion or out-of-band radiation, which could destroy the signal or disturb the adjacent channel. The paper is organized as follow: In section II a short definition of the OFDM and the PAPR to state the main concepts. Then, a brief explanation and description of the system in section III. After that in section III there is some details regarding the clipping and filtering technique. In section IV a simulation results is placed with extended details. Finally in section V, we conclude this paper with some of our observations. 


\section{SINGLE-CARRIER TRANSMISSION}

In Fig1, there is a block diagram for a typical end-to-end single-carrier scheme. This transmission system is contains of a band-limited channel denoted by $h(t)$, the transmit mapper $g_{T}(t)$, the receiver demapper $g_{R}(t)$ and equalizer symbolized by $h^{-1}(t)$. The transmitted symbols $a_{n}$ have a period of T for each symbol and the data rate is presented as $R=1 / T$. The output of the process is shown in the following equations (1) and (2) where the AWGN is denoted by $z(t)$ :

$$
\begin{aligned}
& y(t)=\sum_{m=-\infty}^{\infty} a_{m} g(t-m T)+z(t) \\
& g(t)=g_{T}(t) * h(t) * g_{R}(t) * h^{-1}(t)
\end{aligned}
$$

We made assumption in this paper that the transmitted signal is recovered from the fading and noise effects using the equalizer. Since this assumption is made, the noise in the previous equations is ignored and the noise-free output can be formed as follow:

$$
y(t)=\sum_{m=-\infty}^{\infty} a_{m} g((n-m) T)
$$

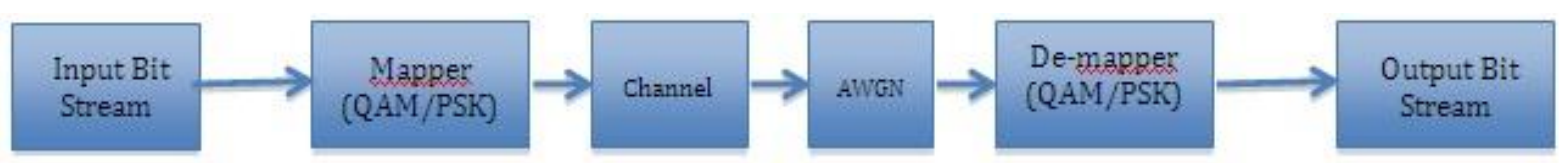

Fig1: Model of single-carrier transmission system.

In the stages of mapping and de-mapping, different modulation techniques could be used. Quadrature Amplitude Modulation (QAM) and Phase Shift Keying (PSK) are used in this paper as a modulation technique. The input sinusoidal signal can be mapped to two components the In-phase component $I(t)$ and the Quadrature component $Q(t)$. To explain the concept of modulation procedure, we can take Binary PSK as an example. In the case of having BPSK, there are two possible values, 0 or 1 . While, if QPSK is used, we would have two binary digits at a time, $(00,01,10,11)$. Table1 show the mapped component represented in terms of $\mathrm{Q}$ and $\mathrm{I}$ :

Table 1: Converting binary digits to complex form.

\begin{tabular}{|l|c|c|}
\hline Binary Digits & $\mathbf{I}(\mathbf{t})$ & $\mathbf{Q}(\mathbf{t})$ \\
\hline $\mathbf{0 0}$ & $1 / \sqrt{2}$ & $1 / \sqrt{2}$ \\
\hline $\mathbf{0 1}$ & $1 / \sqrt{2}$ & $-1 / \sqrt{2}$ \\
\hline $\mathbf{1 0}$ & $-1 / \sqrt{2}$ & $1 / \sqrt{2}$ \\
\hline $\mathbf{1 1}$ & $-1 / \sqrt{2}$ & $-1 / \sqrt{2}$ \\
\hline
\end{tabular}

III. MULTI-CARRIER (OFDM)

To over come the shortages that appear using single-carrier transmission, OFDM is used as a multicarrier transmission [4]. Fig2 shows a block diagram for the OFDM transmission system where it has two main parts: transmitting side and receiving side. The signal gets into the transmitting side starting with mapping process. Then the output of the operation is converted from serial form to parallel form making shorter symbols sequences. A pilot is inserted in between the sub-carrier seeking a way to estimate a start of the OFDM symbols. Afterward the output signal goes through the IFFT operation before adding the Cyclic Prefix (CP). The use of the IFFT is so important to overcome the use of huge number of RF oscillators. The last stage in the receiver is the parallel to serial convertor.

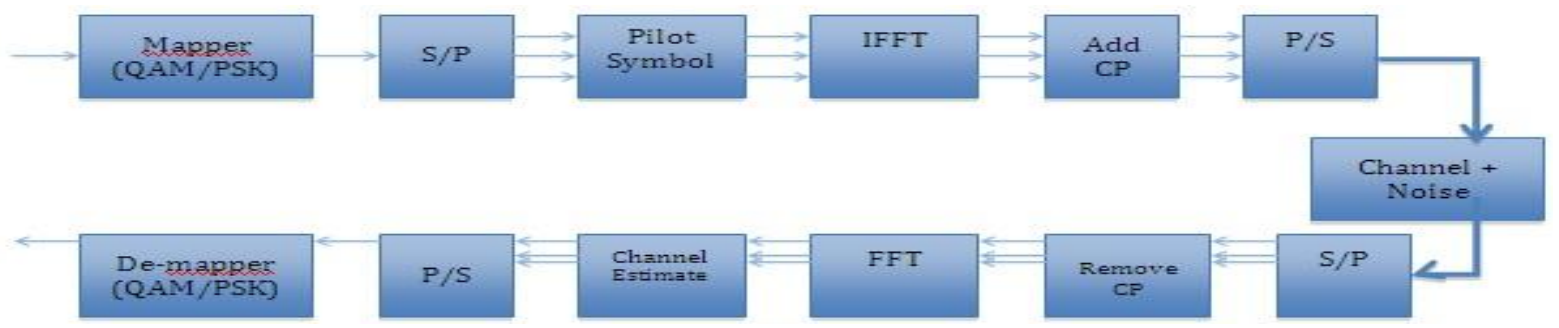

Fig2: Model of Multi-carrier transmission system (OFDM). 
Orthogonality should be achieved to ensure better spectral efficiency for the system and maximum capacity for the transmitting channels. The aim of maintaining orthogonality is to prevent the overlapped of center frequency for the subcarrier and giving a chance for the spectrums of the subcarriers to overlap desiring higher spectral efficiency. Assume there is complex exponential signal $\left\{e^{j 2 \pi f_{k} t}\right\}_{k=0}^{N-1}$ that represents the subcarriers in the OFDM signal while we have $f_{k}=k / T_{\text {sym }}$. To check the orthogonality of any two sub-carriers in the OFDM signal, the following conditions can be applied as shown in equation (4):

$$
\begin{gathered}
\frac{1}{T_{\text {sym }}} \int_{0}^{T_{\text {sym }}} e^{j 2 \pi f_{k} t} e^{-j 2 \pi f_{i} t} d t=\frac{1}{T_{\text {sym }}} \int_{0}^{T_{\text {sym }}} e^{\frac{j 2 \pi k}{T_{\text {sym }}} t} e^{-\frac{j 2 \pi i}{T_{\text {sym }}} t} d t \\
=\frac{1}{T_{\text {sym }}} \int_{0}^{T_{\text {sym }}} e^{\frac{j 2 \pi(k-i)}{T_{\text {sym }}} t} d t \\
= \begin{cases}1, & \text { when } k=i \\
0, & \text { otherwise }\end{cases}
\end{gathered}
$$

If the dot product of the tested signals is not equal zero, we can say that these two signals did not fulfill the orthogonality condition, otherwise the two signals are orthogonal to each other. To prevent ISI, guard intervals are used to be inserted between the OFDM symbols to avoid the overlapping that could be caused by transmitting delay. The used of guard intervals is so important to ensure a symbol full attenuating. There are several types of guard intervals: zero padding, cyclic prefix (CP) and cyclic suffix (CS). In this paper we inserted CP as guard intervals to prevent ISI. On the receiving side, the signal is down converted to get the baseband signal and we make assumption that both side of the system, the receiver and the transmitter, are synchronize in time. After that, the received signal is converted from serial to parallel form to have shorter sequence in length. Then the CP is removed from the signal preparing it to proceed through the FFT operation. In the ideal case, we would have perfect output, which is impossible in practical operations.

\section{PEAK-TO-AVERAGE POWER RATIO (PAPR)}

Depends on the ratio between the maximum power of the complex pass-band signal and the mean power of it, we can calculate the PAPR. The value of PAPR can be calculated as following equation shows [7]:

$$
P A P R=\frac{\max \left(x^{2}(t)\right)}{\text { mean }\left(x^{2}(t)\right)}
$$

Where $x(t)$ is presented in the equation to denote the amplitude of the complex pass-band signal. To calculate the probability of having PAPR greater than the threshold value for the OFDM signal, we could use Complementary Cumulative Distribution Function (CCDF). By simulating the CCDF, we compare the theoretical values with the simulation results and the PAPR keeps increasing when the number of carriers increases. In the simulation part there is a figure showing the CCDFs of OFDM signals with different number of subcarriers.

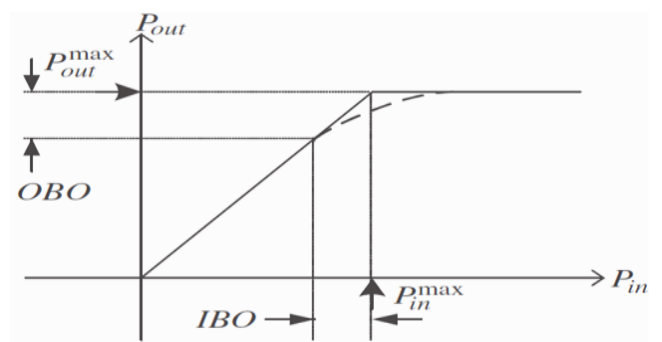

Fig3: Input and output characteristic of an HPA [5].

If we use linear amplifiers that they have larger input than the nominal value, we could have an output with a nonlinear distortion. In Fig3, there is a diagram for High Power Amplifier (HPA) showing the input and output characteristic. In this diagram, the input power is denoted by $P_{i n}$ while the outputs power is represented by $P_{\text {out }}$. To keep the linearity, the maximum output power is limited by the value $P_{\text {out }}^{\text {max }}$ and the maximum input power is pounded by $P_{\text {in }}^{\max }$. Both the input and output power are backed off to insure a linear operation and the area of the backing off is termed by Input Bach-Off (IBO) and Output Back-Off (OBO). The following two 
equations describe the IBO and the $\mathrm{OBO}$ in terms of the input-output power and the maximum input-output power:

$$
\begin{aligned}
& I B O=10 \log _{10} \frac{P_{\text {in }}^{\max }}{P_{\text {in }}} \\
& I B O=10 \log _{10} \frac{P_{\text {out }}^{\max }}{P_{\text {out }}}
\end{aligned}
$$

In the case of having nonlinear HPA characteristic, which is caused by having larger input than its nominal value, we could have out-of-band radiation that makes an overlap between the adjacent signals [8]. Also there is another problem that appears with the nonlinear characteristic of HPA that is the in-band distortion causing a serious issues including attenuation, spinning and offset the received signals.

\section{CLIPPING AND FILTERING}

The clipping is the easiest technique to reduce the power by setting a maximum level for the transmitted signal [9]. Though, this technique has several disadvantages:

- The performance of BER could be affected negatively due to the in-band distortion caused by the clipping.

- Also out-of-band radiation usually appears with clipping technique that could disturb the adjacent channels.

However, we can use filtering operation to decrease the appearance of the out-of-band radiation but the signal may exceed the maximum level of the clipping operation [10]. The block diagram of clipping and filtering technique for PAPR reduction is exposed in Fig4. In this figure, $\mathrm{N}$ denotes the number of subcarrier and $\mathrm{L}$ represents the oversampling factor. In the diagram, The IFFT generate $x^{\prime}[m]$ which is the L-times oversampled signal. As shown in fig4, the FFT-IFFT filter is applied to allow the signal passing through a band-pass filter (BPF) then through a low-pass filter (LPF). The outcome of the filtering stage is a less degraded BER performance and a reduced out-of-band radiation. Though, the PAPR reductions improvements are gained at the cost of regrowth the peak where the signal could go beyond the clipping level after applying the filtering operation.

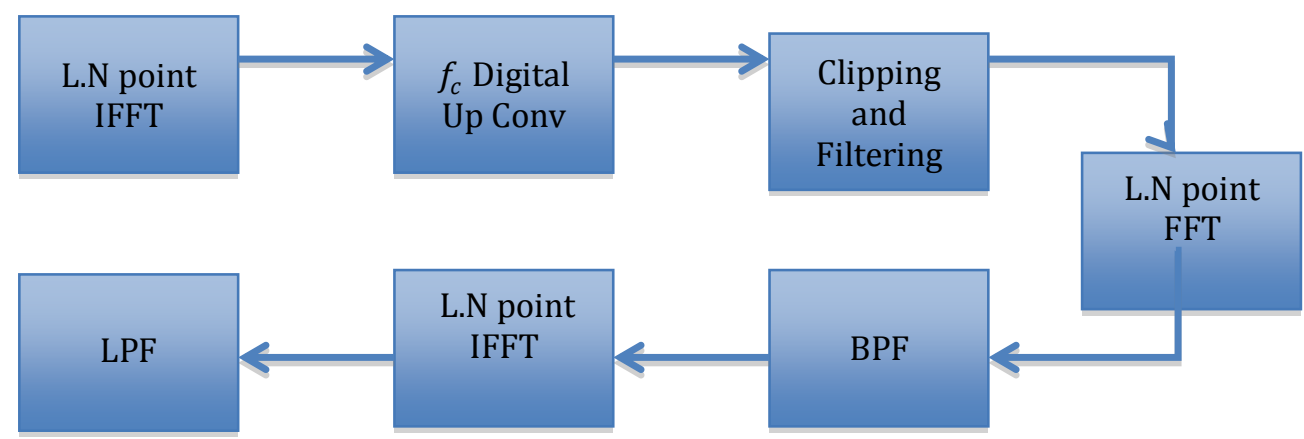

Fig4: The scheme of clipping and filtering technique for PAPR.

The signal $x^{p}[m]$ is the passband modulated one with carrier frequency $f_{c}$. We symbolize the clipped form of the passband-modulated signal as $x_{c}^{p}[m]$. The expression of this signal is shown in following equation:

$$
x_{c}^{p}[m]=\left\{\begin{array}{cc}
-A & x^{p}[m] \leq-A \\
x^{p}[m] & \left|x^{p}[m]\right|<A \\
A & x^{p}[m] \geq A
\end{array}\right.
$$

Where the clipping level is denoted by $\mathrm{A}$ and $(\mathrm{CR})$ is the clipping ratio that can be represented as follow:

$$
C R=\frac{A}{\sigma}
$$

Where the RMS value of OFDM signal is denoted by $\sigma$ and it is well known that $\sigma=\sqrt{N}$ for the baseband and $\sigma=\sqrt{N / 2}$ for the passband OFDM signal. 


\section{SIMULATION RESULTS}

The BER performance is investigated in this comparison using MATLAB code. The definition of the BER can be explained as the effected bits divided by the whole number of the transmitted bits over a time interval [11]. This calculation provides us with a percentage quantity that is a unit-less. In Fig5, there is two curves represent a comparison between the OFDM and the SC transmission systems. The comparison shows the BER performance when we use 4QAM. From the curves we can see that the OFDM transmission system improves the BER percentage compared to the SC. As we stated before, the use of the OFDM transmission has a main drawback, which is the PAPR distribution as shown in Fig6.

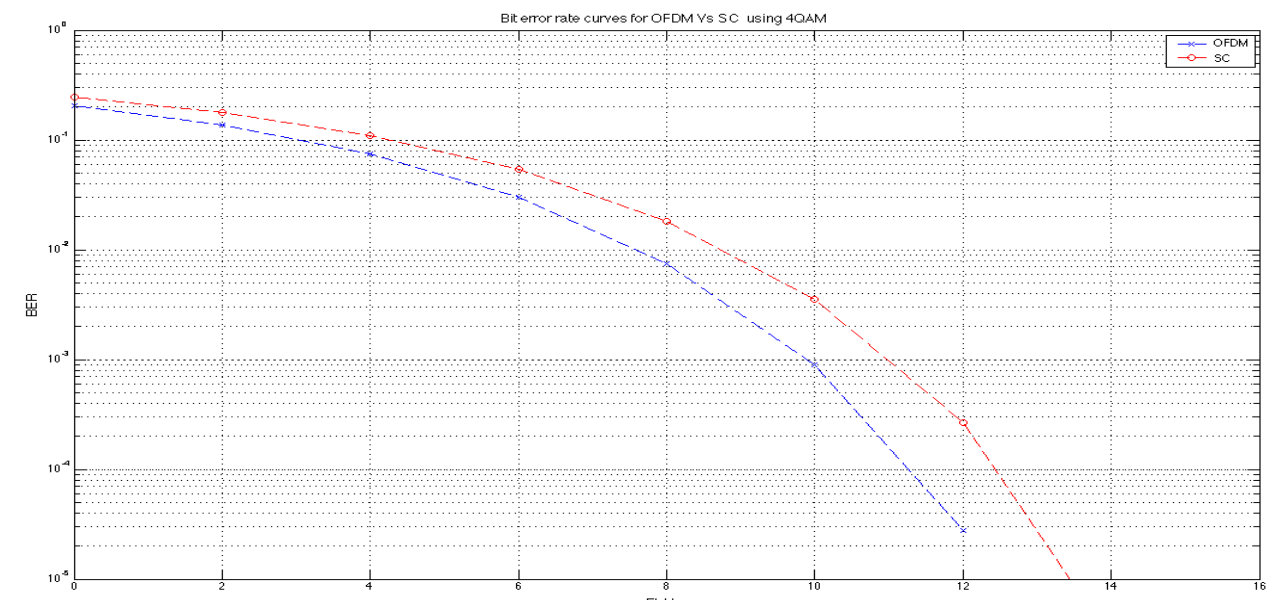

Fig5: BER curves for OFDM Vs. SC using 4QAM.

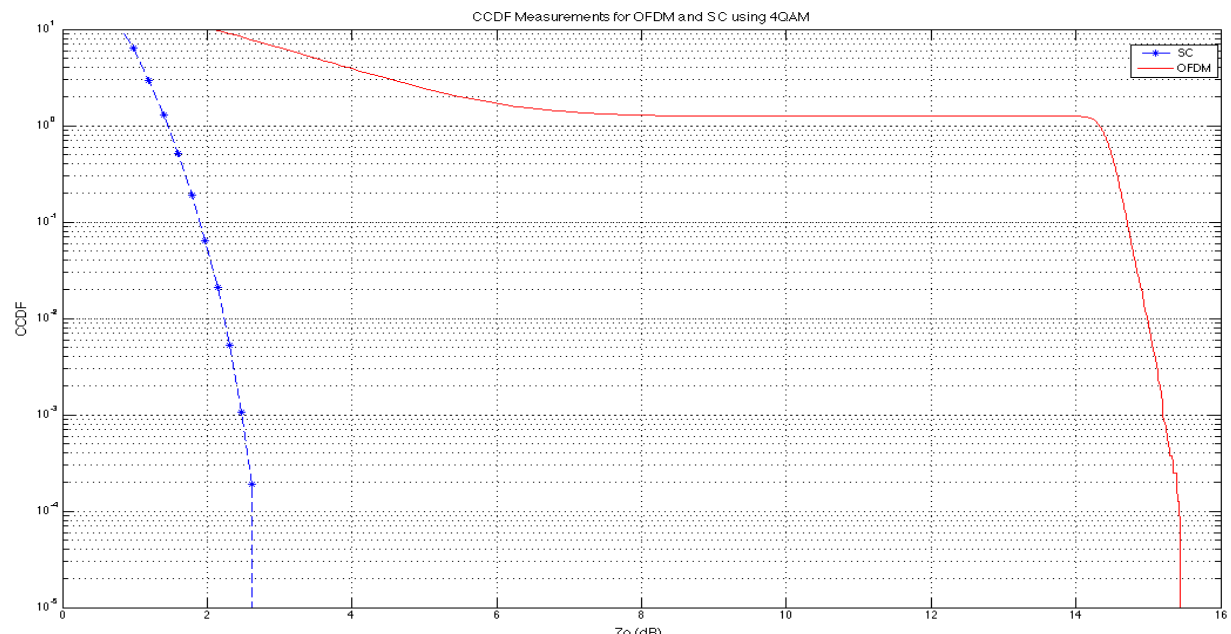

Fig6: CCDFs of OFDM Vs. SC using 4QAM.

Because crest factor $(\mathrm{CF})$ is the square root of PAPR, CCDF of the $\mathrm{CF}$ represents the PAPR distribution. Fig7 shows CCDFs of CF of the OFDM signals after clipping with three different values for clipping ratio. There is an obvious drop in the PAPR of the OFDM signal after clipping. It can be seen as well that the highest PAPR reduction effects happens at the lowest clipping ratio. Fig7 shows that the PAPR is $14.3 \mathrm{~dB}$ for the unclipped signal at CCDF of $10^{-1}$. When we apply a clipping ratio (CR) of 1, PAPR is reduced to $5.2 \mathrm{~dB}$ after applying this algorithm for the same values of CCDF. On the other hand, the BER performance is worsen badly as shown in Fig8 at it gets better when the CR get higher. 


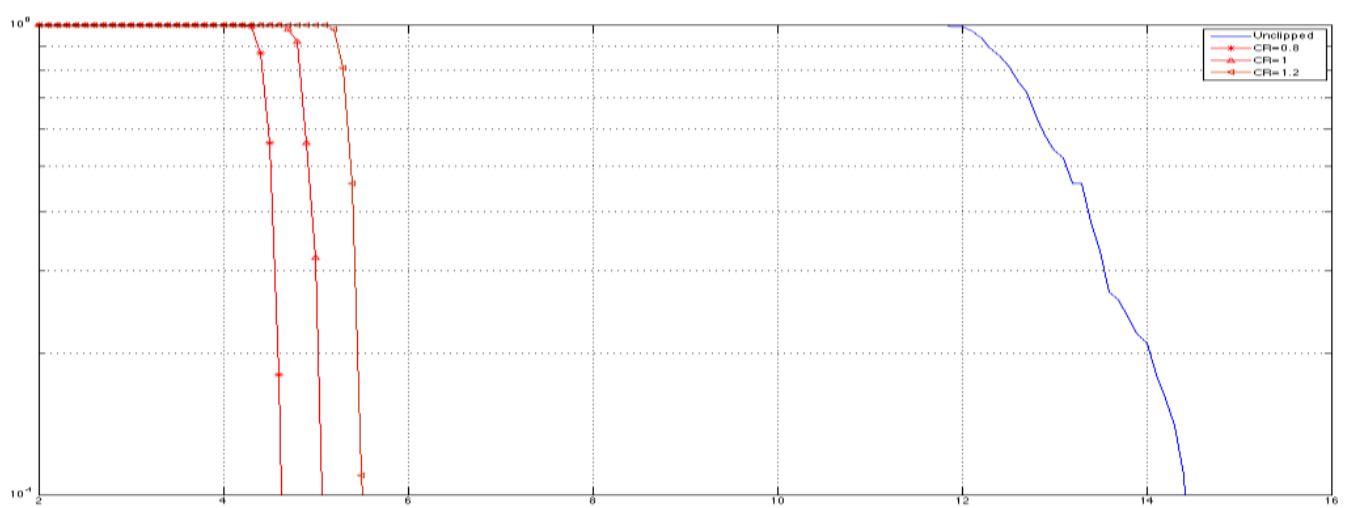

Fig7: PAPR distribution for clipped and unclipped signals.

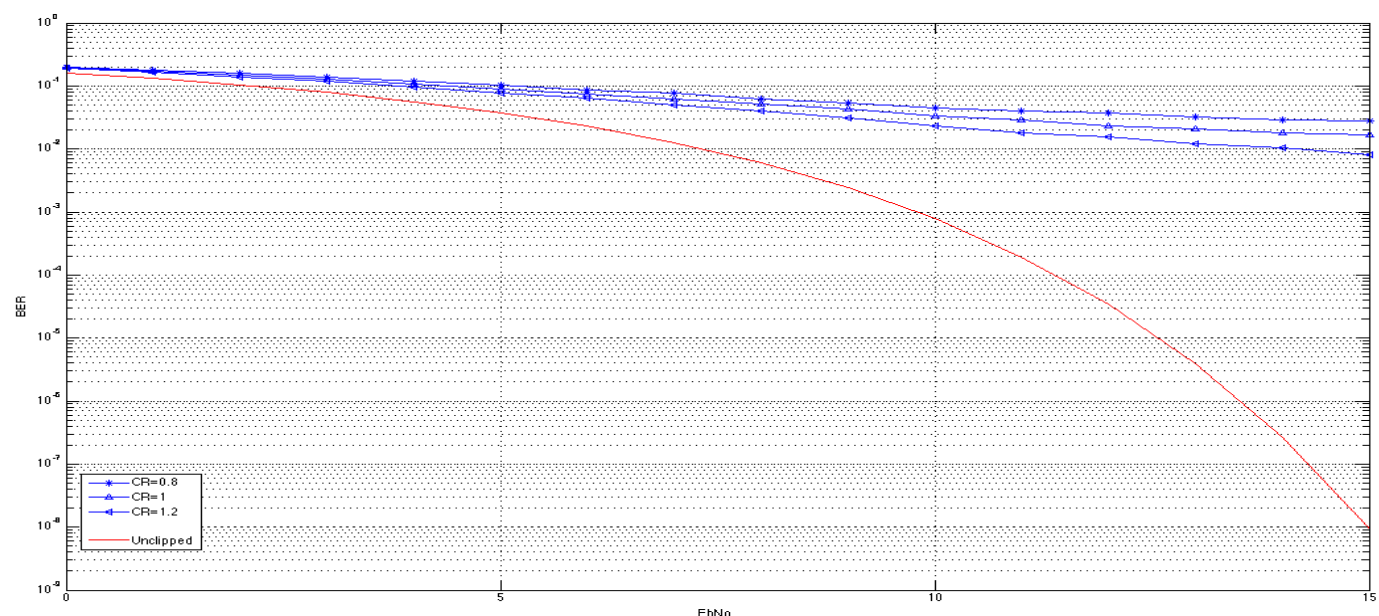

Fig8: BER for clipped and unclipped signals.

Fig9 shows the effect of the clipping and filtering on the PAPR performance where the PAPR is reduce clearly after applying this power reduction technique. The performance of BER for the clipping and filtering technique with respect to the signal to noise ratio (SNR) is compared to the unclipped OFDM signal BER as shown in fig10. Different clipping ratios have been used. It is clear that the performance of the BER get worse as the CR gets lower. The BER of the clipped and filtered signal is 0.0039 at $10 \mathrm{~dB}$ SNR when the clipping ratio is 1.2. The curves also show that the BER of the clipped signal is 0.0019 for the same clipping ratio and SNR.

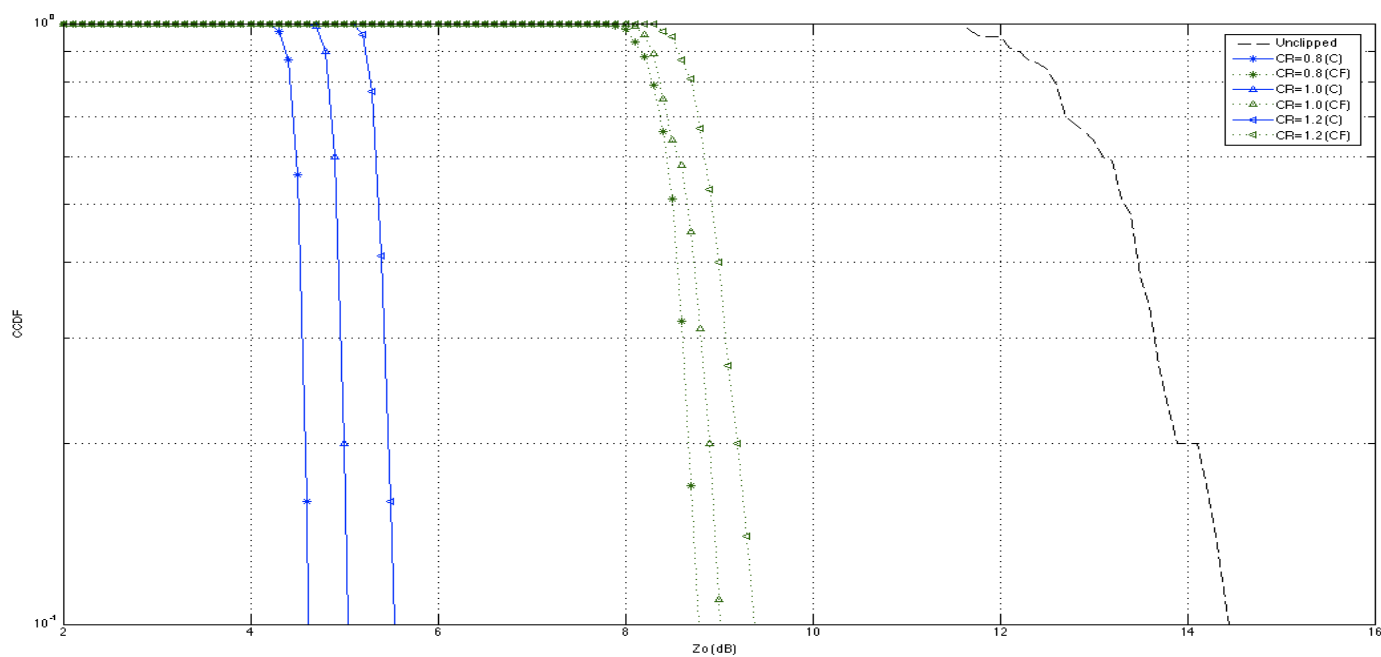

Fig9: PAPR distribution for clipped, filtered, and unclipped signals. 


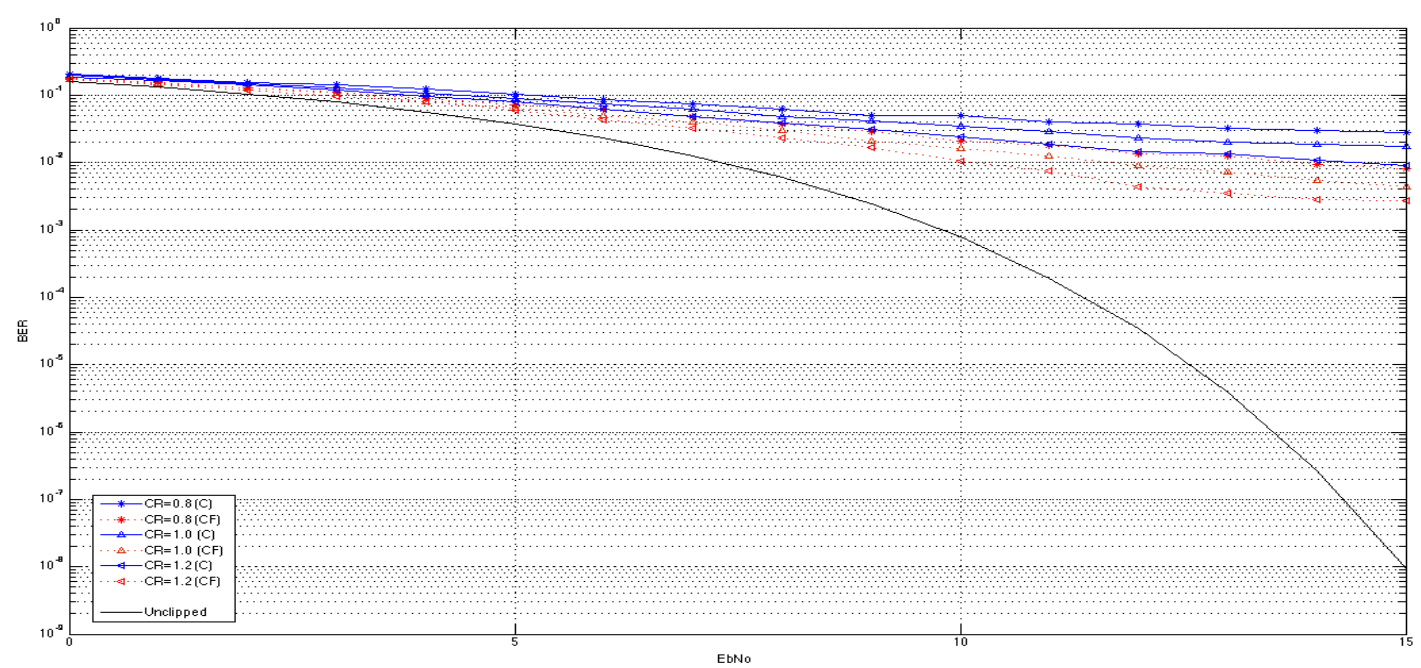

Fig10: BER for clipped, filtered, and unclipped signals.

\section{CONCLUSION}

In this paper, the BER performance is investigated and analyzed for the OFDM and the sing-carrier transmitting schemes to compare between them. For the both techniques, the same parameters are used to make a reasonable comparison and the two signals went through the same channel (AWGN). From the results, we have noticed that the BER performance is improved using the OFDM and outperforms the single-carrier performance. Though, there is a major drawback for using OFDM, which is the high PAPR. This problem can be reduced by using clipping and filtering, as a power reduction technique. The performance of clipping and filtering technique to has been studied in this paper. We conclude from our results that PAPR reduces more at lower CR. The results show how clipping and filtering affect the BER of an OFDM signal and it is clear that the BER is increased after this process. Filter is used to decrease the distortion that result from clipping.

\section{REFRENCES}

[1] A. John, Multicarrier Modulation for Data Transmission: An Idea Whose Time Has Come. IEEE Communications Magazine, 1990.

[2] S. Albdran, A. Alshammari, and M. Matin, The Sensitivity of Bit Error Rate (BER) Performance in Multi-Carrier (OFDM) and Single-Carrier, SPIE optics and photonics, 2012.

[3] J. Tubbax, OFDM versus Single Carrier: A Realistic Multi- Antenna Comparison, EURASIP Journal on Applied Signal Processing, 2004, 1275-1287.

[4] R. Nee, and R. Prasad, OFDM for wireless multimedia communications, Boston, MA: Artech House, 2000.

[5] Y. Cho, J. Kim, W. Yang, and C. Kang, MIMO-OFDM wireless communication with MATLAB (Singapore, Wiley, 2010)

[6] A. Bahai, B. Saltzberg, and M. Ergen, Multi carrier digital communications: Theory and applications of OFDM, Springer Science, New York, 2004.

[7] A. Saleh, R. Valenzuela, A Statistical Model for Indoor Multipath Propagation, IEEE Journal on selected Areas in Communications, 1987, 128-137.

[8] H. Ochiai, and H. Imai, On the Distribution of the Peak to Average Power Ratio in OFDM Signals, IEEE Trans. Commun, 2001.

[9] S. Han, and J. Lee, An overview of peak-to-average power ratio reduction techniques for multicarrier transmission, IEEE Wireless Communications, 2005, 56-65.

[10] B. Alhasson, and M. Matin, Reduction of PAPR for OFDM Downlink and IFDMA Uplink Wireless Transmissions, International Journal of Computer Science and Information Security, 2011.

[11] A. Alshammari, S. Albdran, M.Matin, Study of Bit Error Rate (BER) for Multicarrier OFDM, SPIE optics and photonic, 2012. 